\title{
Efficacy of diphenylcyclopropenone in alopecia areata: a comparison of two treatment regimens
}

\author{
Danuta Nowicka1', Joanna Maj', Alina Jankowska-Konsur¹, Anita Hryncewicz-Gwóźdź²
}

${ }^{1}$ Department of Dermatology, Venereology and Allergology, Wroclaw Medical University, Wroclaw, Poland 2Department of Dermatology, Venereology and Allergology, Faculty of Medicine and Dentistry, Wroclaw Medical University, Wroclaw, Poland

Adv Dermatol Allergol 2018; XXXV (6): 577-581

DOI: https://doi.org/10.5114/ada.2018.77608

\begin{abstract}
Introduction: Alopecia areata (AA) is a skin disease of unclear etiology. In AA, topical immunotherapy with diphenylcyclopropenone (DPCP) is considered the most effective treatment; however, the most common therapies give unsatisfactory results.

Aim: To assess the efficacy of a topical application of a solution of DPCP based on the intensity, duration and number of exacerbations of AA and to compare the efficacy of two treatment regimens.

Material and methods: In this prospective study, 39 patients with AA were enrolled. Group A was treated at weekly intervals and group B at 3-week intervals. Hair loss was assessed by independent dermatologists and documented by photography and dermoscopy.

Results: After 6 months' therapy, hair regrowth greater than 50\% was observed in 21 patients, while worsening, no regrowth, or regrowth of less than $50 \%$ was seen in 18 patients. Regrowth exceeding $50 \%$ of initial loss was observed in 12 of 17 patients with baseline hair loss $<50 \%$, in 9 of 22 patients with severe alopecia, and in 4 of 9 patients with alopecia totalis. Both groups showed significant improvement with higher efficacy in group B (54\%) than group A (46\%).

Conclusions: Treatment at longer intervals may be safer and more comfortable for patients; however, further research is required.
\end{abstract}

Key words: alopecia areata, diphenylcyclopropenone, topical, immunotherapy, prospective study.

\section{Introduction}

Alopecia areata (AA) is a skin disease of unclear etiology although immunologic, genetic, and environmental factors may play an important role in the development of permanent or temporary hair loss. Foci of baldness are usually oval and may be of different sizes. The disease usually affects the scalp. In cases of AA totalis, patients present total loss of hair on the scalp. In cases of AA universalis, patients present the loss of hair over the entire scalp and body, including the scalp, armpits, groin, eyebrows and eyelashes. Clinically, the skin within the affected areas remains normal. In some patients, changes within nails such as regular pitting and ridging, as well as onycholysis, have been observed. Hair regrowth may happen spontaneously or after treatment, but where a treatment-resistant type of the disease is encountered, regrowth is unlikely. Risk factors which may worsen the course of the disease include extensive hair loss (especially AA totalis and universalis), the presence of band-like hair loss (ophiasis), nail involvement, early onset, family history of the disease, and the coexistence of other autoimmune and atopic diseases [1-3].

In the treatment of AA, no gold standard exists. One option suggests the application of glucocorticoids both topically and as injections into the pieces of skin with hair loss. Immunomodulators such as tacrolimus (or less commonly used pimecrolimus), a solution of minoxidil as well as irritant or immunogenic substances are also possible. Ultraviolet A exposure seems to be helpful, too. Systemic treatment of AA is based on medium-dose steroids alone or in combination with immunosuppressants such as cyclosporine A, although this particular agent is related to many side effects and does not guarantee remission [4]. Psychotherapy also plays a role in the treatments of AA, especially in patients who have experienced stressful events, have low self-esteem, or whose course of the disease is associated with stress [5].

Address for correspondence: Danuta Nowicka MD, Department of Dermatology, Venereology and Allergology, Wroclaw Medical University, 1 Chalubinskiego St, 50-368 Wroclaw, Poland, phone: +48 7178423 35, fax: +48 7132709 42, e-mail: danuta.nowicka@umed.wroc.pl Received: 1.09.2017, accepted: 28.09.2017. 
Many treatment regimens for AA include diphenylcyclopropenone (DPCP). The mode of application suggested by Spano and Donovan, among others, is based on the severity of the disease. This regimen is worth recommending in outpatient settings [6]. DPCP is often chosen for treating patients with more than $50 \%$ of hair loss and shows high efficacy. Because of the lack of the recommended regimen of application of DPCP, the authors conducted an experimental treatment comparing two different application regimens for patients with AA.

\section{Aim}

The aim of the study was to evaluate the efficacy of DPCP in patients with AA and to assess whether the efficacy depends on the severity and duration of the disease and the number of exacerbations. Additionally, the study aimed to compare the differences in effects between a weekly application and an every-21-day application.

\section{Material and methods}

The study included 39 patients, 23 women and 16 men, aged $14-1$ years old (mean age: 39.7 years) suffering from AA. Patients were divided into two groups with different treatment regimens based on the topical application of a solution of DPCP. First, a stock solution of 2\% DPCP was prepared by dissolving DPCP powder in acetone. Next, the stock solution was diluted on demand to obtain the following concentrations: $0.00001 \%, 0.0001 \%$, $0.001 \%, 0.01 \%, 0.05 \%, 0.1 \%, 0.5 \%, 1 \%$, and $1.5 \%$ solution. The stock solution was stored in dark bottles until use in low temperature. Due to the risk of evaporation of acetone, DPCP solution was prepared in small portions. The DPCP preparation was administered on affected areas at incrementing doses (starting from $0.00001 \%$ ) until an erythematous reaction lasting for at least 2 days was obtained. The DPCP solutions were applied on the scalp with cotton swabs. Patients were advised to avoid washing the area and protect it from sunlight for $48 \mathrm{~h}$. Group A had applications on day 1, 14 and every 7 days thereafter; group B - on day 1 and every 21 days thereafter. This is the first phase of the prospective study, with results being measured after a 6-month treatment. The next assessments will be conducted after 12 and 18 months.

The diagnosis of AA was made by a qualified dermatologist on a clinical basis. The severity of AA was assessed based on the Severity of Alopecia Tool (SALT) [7]. During the examination, the scalp was divided into four areas in which hair loss was measured (40\% vertex, 18\% right profile, $18 \%$ left profile, $24 \%$ posterior) to achieve a composite score. Next, patients were divided into the following groups according to the disease severity: SO - no hair loss; S1 - hair loss < 25\%; S2 - hair loss of 2549\%; S3 - hair loss of 50-74\%; S4 - hair loss of 75-99\%; and $\mathrm{S} 5$ - total scalp hair loss.
Treatment efficacy was clinically monitored by two independent dermatologists and documented by photography and dermoscopy - DERMLITE CAM (3Gen Inc, San Juan Capistrano, CA, USA).

Informed consent was obtained from all enrolled subjects. The study was conducted in accordance with the Declaration of Helsinki after obtaining approval from the local Bioethics Committee. All subjects gave written informed consent for participation in the study.

\section{Statistical analysis}

Statistical analysis was carried out with Statistica software v10 (StatSoft, Tulsa, OK, USA). The data were presented as means and percentages. Associations between risk factors and treatment results were assessed by Spearman's rank correlation coefficient. The criteria for statistical significance were set at $p<0.05$.

\section{Results}

In the study group, the mean hair loss at baseline was $54.3 \%$. The biggest groups according to SALT were S1, with 8 patients having less than $25 \%$ of their scalp affected, and S5, with 9 patients displaying alopecia totalis. After 6 months of treatment, hair regrowth greater than $50 \%$ was observed in 21 patients, while worsening, lack of regrowth, or regrowth of less than $50 \%$ was seen in 18 patients. In 17 patients with baseline hair loss < 50\%, regrowth exceeding $50 \%$ of initial loss was observed in 12 patients, while it was below $50 \%$ in 5 of them. In 22 patients with severe alopecia (>50\%), regrowth exceeding $50 \%$ occurred only in 9 cases. In 9 patients with alopecia totalis, regrowth exceeding 50\% was observed in 4 cases.

Patients were examined after 6 months of treatment. In 16 patients from group A (weekly applications), worsening, lack of regrowth, or regrowth of less than $50 \%$ was observed in 8 patients, and hair regrowth greater than $50 \%$ was observed in 8 patients. The mean response rate in group A was 46\%. In 23 patients from group B (applications every 21 days), worsening, lack of regrowth, or regrowth of less than $50 \%$ was observed in 10 patients, while hair regrowth greater than 50\% was observed in 13 patients. The mean response rate in group B was 54\%.

In both groups, an association between the size of the affected area and the response to treatment was observed. In patients with baseline hair loss below 25\%, regrowth greater than $50 \%$ was observed in 3 subjects from group A and 4 subjects from group B. In patients with total hair loss at baseline, regrowth greater than $50 \%$ was observed in 2 subjects from each group. Baseline characteristics and results are presented in Table 1.

Results obtained after 6 months of treatment were significantly better than at baseline. Additionally, group B achieved significantly better outcomes than group $A$. The Spearman rank correlation coefficient confirmed that patients with less initial hair loss responded better to 
Table 1. Baseline characteristics and results of treatment

\begin{tabular}{|c|c|c|c|c|c|}
\hline Variable & $\begin{array}{c}\text { S1 } \\
<25 \%\end{array}$ & $\begin{array}{c}\text { S2 } \\
25-49 \%\end{array}$ & $\begin{array}{c}\text { S3 } \\
50-74 \%\end{array}$ & $\begin{array}{c}\text { S4 } \\
75-99 \%\end{array}$ & $\begin{array}{c}\text { S5 } \\
100 \%\end{array}$ \\
\hline \multicolumn{6}{|l|}{ Baseline evaluation: } \\
\hline Initial scalp condition & 8 & 9 & 9 & 4 & 9 \\
\hline \multicolumn{6}{|c|}{ Results in the entire study group: } \\
\hline Regrowth $<50 \%$ & 1 & 4 & 6 & 2 & 5 \\
\hline Regrowth > 50\% & 7 & 5 & 3 & 2 & 4 \\
\hline \multicolumn{6}{|c|}{ Results in group A (weekly applications): } \\
\hline Regrowth $<50 \%$ & 0 & 1 & 3 & 0 & 4 \\
\hline Regrowth > 50\% & 3 & 2 & 1 & 0 & 2 \\
\hline \multicolumn{6}{|c|}{ Results in group B (applications every 21 days): } \\
\hline Regrowth $<50 \%$ & 1 & 3 & 3 & 2 & 1 \\
\hline Regrowth > 50\% & 4 & 3 & 2 & 2 & 2 \\
\hline
\end{tabular}

treatment $(r=0.6)$. Results were not correlated with the length of the disease or the number of exacerbations.

Side effects were minor and transient. In several cases, the transient enlargement of neck lymph nodes was observed. One patient developed a generalized allergic reaction.

\section{Discussion}

A topical solution of DPCP is often used in patients suffering from treatment-resistant $A A$ and in those with more than $50 \%$ hair loss. In the present study, the efficacy of DPCP was confirmed in patients with different severities of AA. Additionally, treatment at longer intervals (21 days vs. 7 days) appeared to give better results and to increase comfort and safety.

The first use of drugs producing contact allergy in the treatment of AA dates back to the 1970s. The mechanism of action of this group of drugs remains unclear. It is probably based on the T-cell response switch from Th1 to Th2 and the reduction of local inflammation around hair follicles induced by inflammatory cytokines related to the Th1-type response [8]. This phenomenon was named by Happle as "antigenic competition" [9]. Among immunologic factors, many reports from the literature describe the CD8 and CD4+ lymphocyte infiltrations in the perifollicular area as most frequently visible in the immune-histological examination in the early stages of the disease. The number of T lymphocytes (especially $T$ helper) in the peripheral blood decreases. On the affected skin, the expression of proinflammatory cytokines such as interleukin (IL)-1 and IL-2, and interferon (IFN)- $\gamma$ along with the TNF receptor I is observed [10]. In the chronic type of the disease, cytotoxic T lymphocytes predominate while the phenomenon of apoptosis, i.e. the process of programmed cell death, increases [11, 12]. Recent stud- ies raised the possibility that $A A$ is not mediated only by the Th1 axis. Mice animal models showed that contact sensitizers producing delayed type hypersensitivity abrogate autoimmune reactions resulting in a reduced number of skin-infiltrating leucocytes and signs similar to blockade of leucocyte extravasation [13], while activation of myeloid suppressor cells contribute to autoreactive $T$ cell silencing [10].

Understanding the multifactorial pathogenesis of AA helps to explain the difficulties in achieving good treatment results. Genetic factors contribute to $10-25 \%$ of AA cases. The Rodriguez et al. study conducted on twins revealed that AA was concordant in $42 \%$ of sets of monozygotic twins and only in $10 \%$ of dizygotic twins [14]. Petukhova et al. in their breakthrough discovery proved the existence of genes significantly associated with AA [15]. Examination of 1,054 patients and comparing them with 3,278 healthy controls allowed for the determination of 8 genes which are significantly involved in the development of the disease. Additionally, it was discovered that some of the genes may be responsible for the development of type I diabetes and rheumatoid arthritis. AA occurs more frequently in patients with Down syndrome. It also often coexists with other immune-mediated diseases such as atopic diseases, acquired vitiligo, lichen planus, Hashimoto's thyroiditis, diabetes mellitus, systemic lupus erythematosus, and rheumatoid arthritis. In some patients, antibodies against various antigens are present in the blood, e.g. thyroid antibodies, thyroglobulin antibodies, antibodies against the parietal cells of the stomach, and nuclear antibodies. Environmental factors, especially stress, trigger the onset or progression of the disease. Many attempts to reveal the association between the development of AA lesions and perceived stress have been undertaken. In the 1950s, Anderson reported that in $23 \%$ of patients suffering from $A A$, the 
onset on the disease had been preceded by some form of mental stress [16]. In lesional skin, several associations were observed: an increased density of innervation and release of an increased amount of substance $P(S P)$ in the perifollicular infiltrate; the increased release of vasointestinal peptide (VIP) and calcitonin gene-related peptide (CGRP-I) in the papillary layer of the skin; and the expression of neuropeptide Y (NPY) on Langerhans cells. Stress stimulates SP release on endings of sensory nerves and next, SP activates a cascade of cellular changes, mast cell degranulation, and the induction of endothelial leukocyte adhesion molecule-1 (ELAM-1) on the vascular endothelium. A predominance of NPY produces a vasoconstricting action, which is responsible for impaired microcirculation in hair follicles. According to some authors, some types of human personality are predisposed to the onset of lesions in the course of AA $[12,17]$.

To date, three contact allergens have been used in the treatment of $A A$ : dinitrochlorobenzene (DNCB), squaric acid dibutylester (SADBE), and DPCP. Due to the mutagenic properties of DNCB and the lack of chemical stability of SADBE, DPCP remains the most frequently used immunomodulator. Treatment with DPCP starts after an initial sensitization with a $2 \%$ solution of this agent. Next, a solution of low DPCP concentration is applied in increasing doses on the skin of the scalp until contact eczema appears. In the literature, the effectiveness of treatment is around $60-70 \%$ with a relatively small frequency of side effects. The majority of researchers opt for a traditional regimen of applications, i.e. 2 weeks following the initial sensitization, the solution is applied every seven days. This regimen was used by Aghaei in 27 patients. In his study, complete remission was obtained in 6 patients and partial remission (10-90\%) in 16 patients [18]. The most frequent adverse events are severe inflammation at the site of application, enlarged neck lymph nodes, and hyper- and hypopigmentation. In several cases, the use of DPCP provoked an outbreak of vitiligo $[19,20]$. In the present study, several cases of transient enlargement of the neck lymph nodes were observed, as well as 1 case of a generalized allergic reaction.

In patients with severe AA, treatment planning is difficult due to a relatively low response rate and limited options. Most of the researchers administer DPCP in weekly applications, but the proper duration of the therapy is unclear. Ohlmeier et al. recommend weekly applications and then, after obtaining complete or cosmetically acceptable re-growth of scalp hair, applications at increasing intervals (2-, 3-, and 4-week intervals). However in their study, the efficacy of maintenance therapy was not assessed. They also recommend continuing DPCP immunotherapy for at least 1 year. Additionally, they found that better outcomes were achieved in patients with less hair-loss at baseline, which is in line with our results [21].

In the present study, the authors proposed a novel regimen of DPCP application. The results indicate that this regimen is more effective than the commonly used weekly applications. It is worth mentioning that this experiment is still being conducted and that patients will be examined again after 12 and 18 months of treatment. The authors hope to shed some light on the effectiveness of the continuation therapy after positive response to DPCP and evaluate the factors that may influence recurrence.

\section{Conclusions}

Treatment of AA based on DPCP may be considered a good therapeutic option, especially in patients with extensive baldness, because of its high efficacy and easy application as well as its rare and mild side effects. Treatment at longer intervals may be more effective, safer and more comfortable for the patients; however, further research is required.

\section{Acknowledgments}

The study was conducted in the Department of Dermatology, Venereology and Allergology, Wroclaw Medical University, Wroclaw, Poland.

\section{Conflict of interest}

The authors declare no conflict of interest.

\section{References}

1. Tosti A, Bellavista S, lorizzo M. Alopecia areata: a long term follow-up study of 191 patients. J Am Acad Dermatol 2006; 55: 438-41.

2. Tan E, Tay YK, Goh CL, Chin GY. The pattern and profile of alopecia areata in Singapore: a study of 219 Asians. Int J Dermatol 2002; 41: 748-53.

3. De Waard-van der Spek FB, Oranje AP, De Raeymaecker DM, Peereboom-Wynia JD. Juvenile versus maturity-onset alopecia areata: a comparative retrospective clinical study. Clin Exp Dermatol 1989; 14: 429-33.

4. Harries MJ, Sun J, Paus R, King LE Jr. Management of alopecia areata. BMJ 2010; 341: c3671.

5. Brzezińska-Wcisło LA, Wcisło-Dziadecka D. Hair diseases: a big problem on a small surface. Postep Dermatol Alergol 2016; 33: 317-22.

6. Spano F, Donovan JC. Alopecia areata: part 2: treatment. Can Fam Physician 2015; 61: 757-61.

7. Olsen EA, Hordinsky MK, Price VH, et al. Alopecia areata investigational assessment guidelines. Part II. National Alopecia Areata Foundation. J Am Acad Dermatol 2004; 51: 440-7.

8. Singh G, Lavanya M. Topical immunotherapy in alopecia areata. Int J Trichology 2010; 2: 36-9.

9. Happle R. Diphencyprone for the treatment of alopecia areata: more data and new aspects. Arch Dermatol 2002; 138: 112-3.

10. Marhaba R, Vitacolonna M, Hildebrand D, et al. The importance of myeloid-derived suppressor cells in the regulation of autoimmune effector cells by a chronic contact eczema. J Immunol 2007; 179: 5071-81. 
11. Herbst V, Zoller M, Kissling S, et al. Diphenylcyclopropenone treatment of alopecia areata induces apoptosis of perifollicular lymphocytes. Eur J Dermatol 2006; 16: 537-42.

12. Ghersetich I, Campanile G, Lotti T. Alopecia areata: immunohistochemistry and ultrastructure of infiltrate and identification of adhesion molecule receptors. Int I Dermatol 1996; 35: 28-33.

13. Zoller M, Freyschmidt-Paul P, Vitacolonna M, et al. Chronic delayed-type hypersensitivity reaction as a means to treat alopecia areata. Clin Exp Immunol 2004; 135: 398-408.

14. Rodriguez TA, Fernandes KE, Dresser KL, Duvic M. Concordance rate of alopecia areata in identical twins supports both genetic and environmental factors. J Am Acad Dermatol 2010; 62: 525-7.

15. Petukhova L, Duvic M, Hordinsky M, et al. Genome-wide association study in alopecia areata implicates both innate and adaptive immunity. Nature 2010; 466: 113-7.

16. Anderson I. Alopecia areata: a clinical study. Br Med J 1950; 2: $1250-2$.

17. Wygledowska-Kania M, Bogdanowski T. Examination of the significance of psychological factors in the etiology of alopecia areata. I. Examining type A behavior. Przegl Lek 1995; 52: 311-4.

18. Aghaei S. Topical immunotherapy of severe alopecia areata with diphenylcyclopropenone (DPCP): experience in an Iranian population. BMC Dermatol 2005; 5: 6.

19. Nilforoushzadeh MA, Keshtmand G, Jaffary F, Kheirkhah A. Diphencyprone induced vitiligo: a case report. Case Rep Med 2012; 2012: 356236.

20. Pires MC, Martins JM, Montealegre F, Gatti FR. Vitiligo after diphencyprone for alopecia areata. Dermatol Res Pract 2010; 2010: 171265.

21. Ohlmeier MC, Traupe H, Luger TA, Böhm M. Topical immunotherapy with diphenylcyclopropenone of patients with alopecia areata - a large retrospective study on 142 patients with a self-controlled design. J Eur Acad Dermatol Venereol 2012; 26: 503-7. 